\title{
La lúdica una estrategia de mediación para eldesarrollo de competencias enel aprendizaje del idioma inglés
}

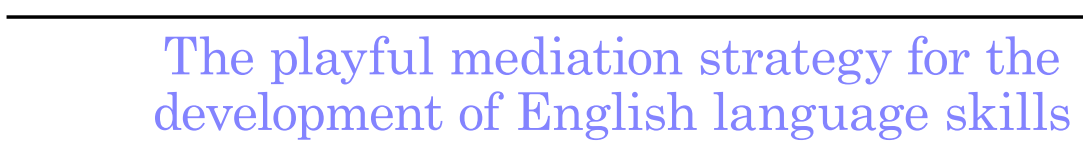

DOI: http://dx.doi.org/10.17981/cultedusoc.9.3.2018.61

Artículo de investigación. Fecha de recepción: 15/06/2018. Fecha de aceptación: 27/11/2018

Mireya Guerra-De La Rosa ${ }^{1}$;

Ledis Elena Vargas-Díaz; Lidis Acosta-Villa; Adriana Sánchez-De Moya; Agapito Hernández-Montero; Amalia Olaya-Correa; Edgar Mendoza-Larios;

Franklin Lubo-Ebrath; Ian Motta-Vásquez; Ingrith De La Cruz-Theran;

Jhon Gómez-Lizcano; Julio Ruiz-Ojeda; Marielis Fuentes; Venus Uribe-Grajales; Yolanda Arevalo-Ascaino y Yomar Camargo-Casadiego ${ }^{2}$

Institución educativa Sierra Nevada de Santa Marta, sede Santa Rosa de Lima (Colombia) mireyadelcarmeng76@gmail.com

Para citar este artículo:

Guerra-De La Rosa, M., Vargas-Díaz, L., Acosta-Villa, L., Sánchez-De Moya, A., Hernández-Montero, A., Olaya-Correa, A., MendozaLarios, E., Lubo-Ebrath, F., Motta-Vásquez, I., De La Cruz-Theran, I., Gómez-Lizcano, J., Ruiz-Ojeda, J., Fuentes, M., Uribe-Grajales, V., Arevalo-Ascaino, Y. y Camargo-Casadie, Y. (2018). La lúdica una estrategia de mediación para eldesarrollo de competencias enel aprendizaje del idioma inglés. Cultura. Educación y Sociedad 9(3), 513-520. DOI: http://dx.doi.org/10.17981/cultedusoc.9.3.2018.61

\section{Resumen}

Actualmente los ambientes de aprendizaje que contemplan la lúdica y la recreación como estrategia de aprendizaje se muestran como ejes fundamentales para la adquisición de mejores y nuevos saberes. Se buscaba implementar una estrategia lúdica para enseñar el idioma inglés, en los estudiantes de primaria de la Institución Sierra Nevada de Santa Marta.La metodología empleada se orienta desde la investigación cualitativa; la unidad de análisis estuvo conformada por 40 estudiantes entre las edades 8 y 9 años; Dentro de los hallazgos encontrados se puede inferir que un $95 \%$ de los estudiantes se sintieron cómodos y con mayores resultados académicos en el área de inglés cuando se implementación de estrategias lúdicas para el aprendizaje de la segunda lengua. Por lo que se puede concluir que los estudiantesse muestrancon mayor interés y aprenden con mayor facilidad cuando seaprende en ambientes donde se encuentre inmersa la lúdica y la recreación.

Palabras clave. Lúdica, estrategia, aprendizaje, inglés.

\section{Abstract}

Currently, learning environments that contemplate leisure and recreation as a learning strategy are shown as fundamental axes for the acquisition of better and new knowledge. The aim was to implement a playful strategy to teach the English language among the elementary students of the Sierra Nevada de Santa Marta Institution. The methodology used was from qualitative research, 40 students were taken as a sample; Among the findings found, it can be inferred that $95 \%$ of students felt comfortable through the implementation of play strategies for learning the second language. So we can conclude that students are more motivated to learn in environments where play is immersed and recreation.

Keywords. Playful, Playful, competitions, mediation, learning

1 Líder del grupo de investigación "Maestros Entusiastas del Ingles".

2 Docentes pertenecientes a la institución educativa Sierra Nevada de Santa Marta, sede Santa Rosa de Lima.

- The author; licensee Universidad de la Costa - CUC.

Cultura, Educación y Sociedad vol. 9 no. 3, pp. 513-520. Diciembre, 2018

Barranquilla. ISSN 2389-7724 Online 


\section{Introducción}

Los procesos de enseñanza del idioma inglés han estado ligado por tiempos a procesos y estrategias convencionales que no viabilizan la interiorización de este proceso, en razón que no generan motivaciones ni interés en los estudiantes, en razón a lo antes expuesto, es de relevancia desarrollar desde el currículo acciones que puedan permitir en los estudiantes el empoderamiento de este aprendizaje.

Actualmente, frente al contexto globalizado que ha impactado el sistema de educación, y frente al mundo actual caracterizado por la comunicación intercultural, por el creciente ritmo de los avances científicos-tecnológicos y por los procesos de internacionalización, han llevado a las instituciones educativas de carácter público a la necesidad de fortalecer el manejo de un idioma común que le permita a la sociedad acceder a este nuevo mundo globalizado, de cambios frecuente en la forma en que nos comunicamos y en la constante búsqueda de desarrollo de competencias que influyen en todos los sectores del saber ser y el saber hacer, específicamente en el área de lenguaje, en el mismo sentido, para Samper y Maussa (2014) un ciudadano de la aldea global debe asumir competencias para la productividad; la ciudadanía plena implica un ser productivo y autorrealizado, que se siente competente y apto para comunicarse y relacionarse en los entornos globalizados y para Arrieta (2016) el educador debe concebir el mundo para sí mismo y para sus educandos desde la idea de la República Universal, es decir, desde un mundo interconectado en el que sus ciudadanos puedan interactuar sin ningún tipo de restricciones. En ese orden de ideas,surge la lúdica como una estrategia que permea toda posibilidad para generar aprendizajes motivadores y de interés. .

La Institución Educativa Departamental Agropecuaria Sierra Nevada de
Santa Marta, busca cambiar la realidad presentada en esta comunidad educativa formando niñas, niños, jóvenes que sean capaces de enfrentar y aportarle, a una sociedad globalizada y que crece a pasos agigantados en ciencia y tecnología. Donde la articulación curricular de las diferentes áreas del saber se constituye como eje fundamental para la adquisición y manejo de un segundo idioma; teniendo en cuenta que en el currículo se establecen los contenidos y ejes fundamentales para que el estudiante desarrolle un proceso de enseñanza-aprendizaje efectivo (Duno, Marín, Luque, 2008).

No obstante, los resultados que muestran las pruebas saber en inglés como en otras áreas, son desalentadoras. Las razones se fundamentan en el decrecimiento de la institución, donde anteriormente, la misma contaba con 30 sedes a lo largo y ancho de la imponente Sierra Nevada de Santa Marta, sin embargo, debido a la presión de los habitantes de la parte alta de la Sierra Nevada, quienes gestionaron la división de las sedes, la institución logró solo mantener 11 escuelas pertenecientes a la sede principal; sedes que carecen de docentes requeridos para cubrir las necesidades, obligados a atender todas las áreas del currículo sin poder profundizar en su área de profesión.

Así mismo, el bajo rendimiento académico y poca motivación de los estudiantes para la adquisición del aprendizaje, son elementos trascendentales en la obtención de esos resultados, pues las habilidades al comprender, escribir, leer y hablar el inglés han sido poco desarrolladas.

Partiendo de la técnica de observación y los resultados de las pruebas académicas del Instituto Colombiano para el Fomento de la Educación Superior (ICFES) de la Institución Educativa Departamental Agropecuaria (IEDA)

Sierra Nevada de Santa Marta, se puede apreciar que los estudiantes no tienen dominio a nivel de gramática y de expre- 
sión oral del idioma inglés, desconocen vocabulario, estructuras, expresiones y modismos del inglés.

Teniendo en cuenta las exigencias sociales del entorno social y educativo frente al dominio del idioma inglés y frente a todas las áreas del saber en fin de fortalecer la calidad educativa y calidad de vida de los estudiantes; se hace necesario formar al estudiante de manera integral, con pertinencia social, con la atención a la globalización y a las tecnologías y, de manera especial, a en la formación ética y crítica de los mismos (Inciarte, Canquiz 2009). Para lograr esto, se deben formar estudiantes competentes, y así, lograr ciudadanos con la capacidad de establecer conversaciones en inglés, de tal forma que puedan insertar al país en los procesos de comunicación universal, con estándares internacionalmente (Ministerio Nacional de Educación, s.f.).

Lo anterior establece que los estudiantes deben ascender al nivel B1, el mínimo establecido por el Ministerio de Educación Nacional (MEN), deberán superarlo, para lograr obtener un nivel más avanzado: B2, que será exigido en las pruebas ICFES y Exámenes de Calidad de Educación Superior(ECAES), por los ya nombrados entes reguladores de la Educación en Colombia. Debido a esto, las estrategias de enseñanza y aprendizaje deben atinar a formar estudiantes capaces de afrontar los retos y exigencias que la sociedad requiere, una de estas necesidades es el dominio del inglés, considerado el lenguaje universal, es por esto, que los procesos deben ser bien orientados y atractivos para los educandos y garantizar así la formación de seres humanos competitivos.

A lo largo de los años se han venido realizando estudios sobre las dificultades que presentan los estudiantes en el aprendizaje del idioma inglés en instituciones de diferentes ciudades y países en las que se encuentra esta problemática. Zapata (2011), lo que ha generado la necesidad de realizar esta investigación con el objetivo de diseñar estrategias que favorezcan la enseñanza del idioma inglés en los estudiantes de $4^{\circ}$ de la IEDA Sierra Nevada De Santa Marta ubicada en una zona rural del municipio de Fundación llamada Santa Rosa de Lima.

En la búsqueda de alternativas y propuestas enfiladas a solucionar esta problemática, surge la lúdica como una estrategia que ofrece herramientas motivantes, constructivas, significativas, integradoras y formativas que garantizan la eficacia de los procesos de enseñanza y aprendizaje (Monsalve, Foronda y Mena, 2016). Es por esta razón, que un docente de inglés debe tener la capacidad de transformar una clase simple, monótona y rutinaria en una experiencia maravillosa e inolvidable para los niños, utilizando la lúdica como estrategia pedagógica, llevándolos a participar de manera activa y divertida en juegos, dinámicas, competencias, canciones, juegos de rol (role plays), acordes a su grado y edad escolar que le permitan adquirir un aprendizaje significativo del idioma inglés.

Según el Ministerio de Educación Nacional (2006), Emplear estrategias para fomentar el aprendizaje de una lengua extranjera en los estudiantes es la oportunidad para el desarrollo social, cultural y cognitivo de los estudiantes ya que esta permite:

a. Fomenta el respeto y la valoración de la pluralidad y de las diferencias, tanto en el entorno social que los rodea como en mundo externo.

b. Apoyar a los estudiantes en la práctica de la interacción social y en la negociación cultural de aprendizajes. Así mismo, mejora su capacidad para entablar relaciones con otras personas y para desenvolverse en situaciones nuevas.

c. Desarrollar a nivel cognitivo la capacidad lingüística en el individuo. 
Además de todas las oportunidades que se abren con el manejo de una lengua extranjera, el inglés se ha convertido en una herramienta muy importante para la educación la cual facilita el intercambio de conocimientos y experiencias con otros países cuya lengua oficial es diferente al inglés, porque permite la comunicación a través de un idioma común y difundido; es importante que el aprendizaje de una lengua extranjera sea ante todo práctico; por lo tanto, las actividades deben ir encaminadas a reflejar la propia realidad del alumno(a), su entorno más inmediato y las actividades que realiza a menudo, en la institución este proceso normalmente se da en un aula (Moreno, 2010).

La actitud del maestro (a) es básica para transmitir la actitud positiva, el gusto por la nueva lengua y la superación diaria en su aprendizaje He aquí razones por las cuales vale la pena aprenderlo (Castaño, 2010).

A continuación se presentarán los aspectos conceptuales que se tomaron en cuenta para el desarrollo de la investigación.

\section{El rol educativo de la lúdica}

La lúdica ha sido entendida como una dimensión inherente al ser humano y propia del mismo, esta se encuentra incluida en la mayoría de las actividades que el hombre realiza y desarrolla a lo largo de su existencia. Es importante mencionar, que un niño, que se desarrolla bajo ambientes y momentos lúdicos será un ser que se integre fácilmente a la sociedad, será más comunicativo, creativo, aceptará normas y aprenderá a aceptar la derrota el fracaso y también aceptar el hecho de ganar. En otras palabras, será una persona capaz de afrontar los retos que se le presenten en su diario vivir (Ballestero, 2014).

Es necesario también aclarar la diferencia entre lúdica y juego. Es común para muchas personas pensar que estos conceptos se refieren a lo mismo. Los juegos son una manifestación lúdica y la lúdica no se limita sólo a juegos, va más allá, trascendiéndolos, con una connotación general, mientras que el juego es más particular. Entendiendo lo anteriormente expuesto podemos decir, que la lúdica ofrece una amplia gama de actividades, el juego sería sólo una de ellas, partiendo de esto, el baile, la pintura, el canto, el arte, la poesía, la escritura, y muchas más son manifestaciones lúdicas (Posada, 2014).

La lúdica sin lugar a dudas, se ha convertido en una herramienta fundamental para promover el desarrollo de competencias en los procesos de enseñanza- aprendizaje, puesto que lo dinamizan y ofrece un sinfín de actividades que se tornan motivantes, divierten y pueden convertir una clase con objetivos claros y bien definidos en una experiencia única e irrepetible, que sin lugar a dudas redundará en beneficios no sólo de tipo cognoscitivo, sino de carácter formativo e integral que activan la creatividad, imaginación y la fantasía (Ballesteros, 2014).

\section{Factores asociados al Aprendizaje de la Lengua Extranjera}

El proceso de conocimiento de una lengua extranjera implica el desarrollo una serie de procesos cognitivos y competencias comunicativas como la comprensión e interpretación, diferentes estudios han comprobado que el aprendizaje de una o varias lenguas extranjeras, no perjudica la lengua nativa, por el contrario, el lenguaje materno si fue adquirido de manera correcta contribuirá de manera significativa a la adquisición del conocimiento del idioma extranjero, mediante el uso de las estrategias. Es importante saber y dejar en claro, que hay muchos factores que inciden en el aprendizaje de una lengua extranjera como su edad de iniciación, mientras más temprano se de este proceso de aprendizaje más sencillo será la adquisición de su conocimiento (Ministerio Nacional de Educación, s.f.).

La enseñanza de un segundo idioma desde la primaria es obligatoria en todas las instituciones de Colombia de hecho la Ley General de Educación ordena 
su introducción. El objetivo principal del aprendizaje de una lengua extranjera en Colombia es que los estudiantes tengan la capacidad de comunicase de una manera eficaz y fluida; para el aprendizaje de la lengua extranjera es importante la construcción de saberes, la interacción social, interacción con los elementos de aprendizaje y lo más importante habilidades lingüísticas para poder comunicarse en una lengua distinta a la materna(Ministerio de Educación Nacional, s.f.).

\section{Metodología}

El estudio se desarrolló con un abordaje metodológico desde el paradigma empírico analítico, a partir del método cualitativo; El alcance de la investigación es descriptiva, por lo que permite identificar características del objeto de estudio, señala formas de conducta y actitudes del universo investigado, establece comportamientos concretos y descubre y comprueba la asociación entre categorías de investigación (Martínez, 2006).

\section{Participantes}

Se tomó como unidad de análisis los niños y niñas entre las edades de 8 y 9 años del cuarto $4^{\circ}$ grado de primaria de la IED Sierra Nevada de Santa Marta, sede Santa Rosa de Lima ubicada en el departamento del Magdalena. Se realizó un muestreo no probabilístico de tipo intencional, donde se seleccionaron 40 participantes; como criterio de inclusión se tuvo en cuenta el grado escolar, rendimiento académico y la edad. El estudio cumplió con los estándares éticos de solicitud del consentimiento informado a nivel institucional.

\section{Técnicas e Instrumentos}

Para la recolección de la información teniendo en cuenta metodología de la investigación fue seleccionada la encuesta, según Cruz y Carmona (2014), es una técnica de investigación que se aplica a una muestra de una población específica para recoger información de algunas creencias, opiniones y actitudes de las personas. Con el propósito de identificar normas y patrones de comportamiento para luego conocer las relaciones entre acontecimientos

El diseño de la encuesta enfatizó en obtener información exacta y precisa con respecto al propósito de la investigación, descubriendo la información respecto a los sujetos de estudio con relación a como sentían los estudiantes con la implementación de las estrategias plateadas en el proyecto, haciendo una comparación sobre las clases tradicionales y las clases con la implementación de estrategias lúdicas con el componente investigativo. Esta encuesta fue aplicada a los 40 estudiantes participantes de la muestra con el apoyo del grupo de docentes.

El instrumento consta de 5 ítems y que requiere de los siguientes datos de identificación edad, cursos, estrato y sexo.

Teniendo en cuenta que los niños que realizarían la presente encuesta se encuentran en edades entre los ocho (8) y nueve (9) años se realizaron preguntas de fácil entendimiento, con el objetivo que los estudiantes pudieran comprender fácilmente las preguntas dando de esta manera una respuesta clara y sincera.

\section{Resultados}

A continuación, se presentan los principales hallazgos de la encuesta realizada a los estudiantes posterior a la implementación de nuevas técnicas y metodologías lúdicas, para la enseñanza del idioma inglés basándose en los componentes contextuales y culturales, realizando un proceso vivencial desde su cotidianidad, esto con el fin de identificar las percepciones de los estudiantes con las actividades desarrolladas. 


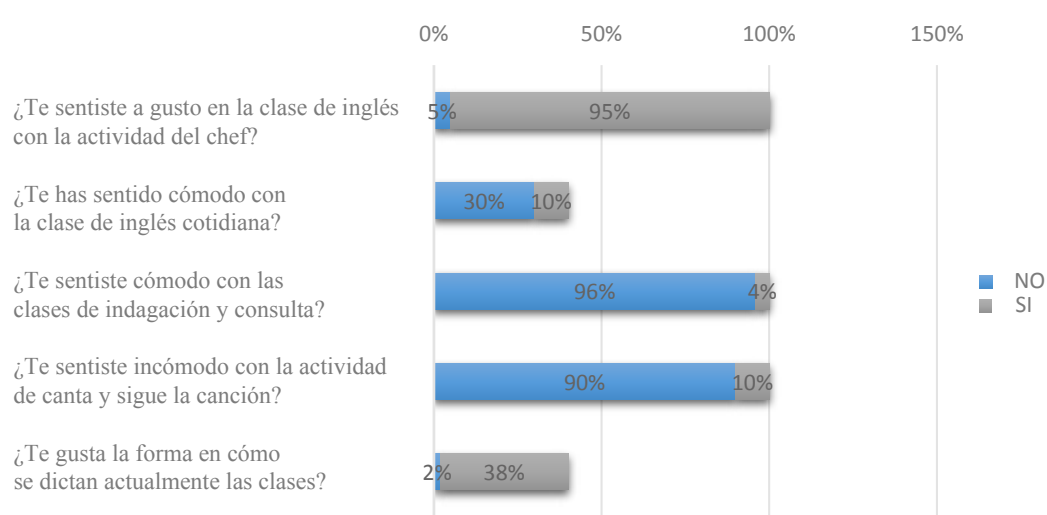

Figura 1. Percepción de la Clase de Inglés.

Fuente:elaboración propia.

De acuerdo con los resultados de la encuesta con respecto a la primera pregunta que consiste en una actividad lúdica práctica, donde los estudiantes respondieron si sentían a gusto con las nuevas actividades a partir del ejercicio del chef, realizada a partir del juego y la lúdica, se encontró que el $95 \%$ de los estudiantes se sintieron cómodos al momento de realizar la actividad en la que debían exponer en ingles los ingredientes de su comida favorita con la ayuda y la instrucción de la profesora. Esto nos indica así como señala Majo y Marqués (2001), que a través del juego se pueden construir herramientas operativas que brindan amplias posibilidades a la práctica educativa como un elemento renovador de la enseñanza y como medio para el aprendizaje que posibilita el desarrollo integral del niño.

Con respecto a la segunda pregunta el $90 \%$ de los estudiantes manifiestan sentirse incomodos con la dinámica de las clases anteriores a la implementación de la lúdica, esto nos muestra que la lúdica, sin lugar a dudas, se convierte en una herramienta fundamental para promover el desarrollo de competencias en los procesos de enseñanza- aprendizaje, ya que dinamiza la práctica del docente en torno a las necesidades que presentan los estudiantes en la forman que aprenden y procesan la información (Ballesteros, 2014).

En las actividades de indagación y de consulta el $96 \%$ de la población no se sintió cómodo, expusieron que era difícil, ya que viven en una zona de difícil acceso a internet, con dificultades para acceder a libros y lo describen como una actividad aburrida; en el cuarto ítems se evaluaba la actividad implementada en la cual una población del 90 \% se sintieron cómodos, exponiendo que era divertido el otro $4 \%$ de la población expusieron, que no se sintieron cómodos debido al temor a de exponerse al público; en los dos últimos ítems se hizo una comparación entre la forma en cómo se dictaban las clases anteriormente y como se dictan actualmente implementando las estrategias lúdicas, donde solo el $4 \%$ de los estudiantes, se sentía cómodo con la manera en cómo se estaban implementando las clases anteriormente, mientras que el 98\% de los estudiantes, respondieron que si se sentían cómodos y felices de aprender el idioma con la implementación de dinámicas que facilitaban la adquisición de aprendizajes. 


\section{Discusión}

Partiendo de los hallazgos derivados de la investigación realizada, se pueden determinar los siguientes:

Surge la necesidad de replantear la práctica pedagógica del maestro, innovando el proceso de enseñanza, articulando los contenidos de las diferentes áreas del saber y específicamente en el área de inglés, con actividades lúdicas que despierten el interés de los estudiantes por aprender, que se interesen por los procesos académicos en torno al desarrollo de competencias, científicas e investigativas que posibiliten mayores y mejores resultados que tiendan a mejorar la calidad educativa. En este sentido se hace necesario trabajar de la mano con herramientas y estrategias basadas en las TIC, puesto que incrementan la iniciativa y motivación de los estudiantes en relación a la apropiación de conocimiento frente a sus compromisos escolares (Avendaño y Martínez, 2013).

Se hace necesario el abordaje o profundización del área de inglés como un área fundamental del currículo institucional, pues el aprender otra lengua como señala el MEN, (2006) favorece el desarrollo de un mayor grado de conciencia metalingüística y aumenta la habilidad para apreciar lo arbitrario y lo convencional de los símbolos lingüísticos. Según Franco, Blanco y Cortes (2013), estas habilidades tienen que ver con la capacidad para describir y analizar el sistema lingüístico. Los sujetos pueden analizar, pensar, reflexionar sobre la forma, el contenido o el uso del lenguaje en diferentes contextos.

Se busca formar un estudiante, con competencias lingüísticas que le permitan proyectarse a nivel internacional. Capaz de dar respuesta a los cambios dados por la globalización de manera eficiente, comprometidos con un proyecto de vida, que incluye un cambio en su entorno social, con conciencia crítica, con la realidad socioeconómica, política y cultural de su contextos, que trabaja en su crecimiento personal pero en constante búsqueda de un desarrollo local.

Se hace evidente la necesidad de implementar estrategias lúdicas que lleven al estudiante a curiosear, a indagar, a explorar, a generar procesos de autoformación, que les faciliten adquirir nuevos saberes en torno al manejo de una segunda lengua. Cabe resaltar, que la adquisición de estos saberes solo es posible cuando se forma al estudiante de manera integral, trabajando de manera paralela con los distintos contenidos curriculares. MEN (2006). En este mismo orden de ideas, se recomienda el establecimiento de diseños curriculares flexibles capaces de adaptarse a los cambios continuos presentes en la sociedad actual, donde los avances tecnológicos marcan la pauta y las necesidades sociales la demanda, para las actuaciones presentes $\mathrm{y}$ futuras.

Finalmente estos planteamientos confirman que los estudiantes del IEDA Sierra Nevada De Santa Marta se muestran con mayor disposición y motivación por aprender un segundo idioma en ambientes donde se encuentre inmersa la lúdica y la didáctica, implementando estrategias innovadoras desde el uso de las TIC y la investigación como elementos fundamentales en la adquisición de conocimientos y en la creación de ambientes de aprendizajes dinámicos; desde las diferentes áreas se aplicaron estas estrategias, obteniendo como resultados un incremento en los niveles de producción, participación reflejadas en las notas definitivas del periodo académico. De acuerdo con los objetivos planteados inicialmente en el proyecto de investigación, podemos concluir que a cabalidad se logró diseñar y aplicar las estrategias lúdicas para el aprendizaje del idioma inglés en los estudiantes de primaria de la Institución Sierra Nevada de Santa Marta. 


\section{Referencias}

Arrieta, M. (2016). Los masones en el mundo: Geopolítica masónica. La historia de los hijos de la Luz. Córdoba: Almuzara.

Ballesteros, A. (2014). Uso de las actividades lúdicas en la enseñanza del inglés [Tesis de Pregrado]. Universidad de Valladolid, Valladolid.

Castaño, C. (2010). La lúdica como recurso para la enseñanza del inglés en grado primero. [Tesis de pregrado]. Universidad de la Amazonia, Florencia, Colombia. Recuperado de https://edudistancia2001. wikispaces.com/file/view/25.+LA+L\%C3 $\% 9 A D I C A+C O M O+R E C U R S O+P A R A+$ LA+ENSE\%C3\%91ANZA+DEL+INGL\% C3\%89S+EN+EL+GRADO+PRIMERO. pdf

Cruz, M. y Carmona, M. (2014). Competencias argumentativas en estudiantes de educación superior. Revista Internacional de Ciencias Sociales y Humanidades SOCIOTAM, 24(2), 115-137.

Duno, D., Marín, F. y Luque, M. (2008). Enseñanza de las ciencias básicas integradas fundamentadas en el desarrollo del pensamiento. Multiciencias 8. 185191.

Franco, M. (2011). Intervención psicoeducativa para la promoción de la comprensión lectora. Cultura, Educación y Sociedad, 2(1). 75-84.

Inciarte G, y Canquiz, R. (2009). Una concepción de formación profesional integral. Revista de Artes y Humanidades UNICA, 10(2). 38-61.

Moreno, H. (2010). Plan de Estudios Lengua Extranjera: inglés. Bogotá, D. C.: Ediciones S E M.
Monsalve, M., Foronda, R. y Mena, S. (2016). La lúdica como instrumento para la enseñanza-aprendizaje. [Tesis Especialización]. Fundación Universitaria Los Libertadores, Medellín.

Marqués, P. y Majo, J. (2002). La revolución educativa en la era Internet. Barcelona: CissPraxis.

Montenegro, M., Blanco, M. y Cortes, O. (2013). Papel de las habilidades metalingüísticas en los procesos de lectura y escritura en la educación superior. Escenarios, 11(2), 82-86.

Posada, R. (2014). La lúdica como estrategia didáctica. [Tesis magister]. Universidad Nacional, Bogotá, D:C., Colombia Recuperado de http://www.bdigital. unal.edu.co/41019/1/04868267.2014.pdf

República de Colombia. Ministerio de educación. (2012). Estrategias y metodologías pedagógicas. Cucutá: Proyecto "Quedate". Recuperado de http:// www.ufps.edu.co/ufpsnuevo/archivos/110_2013.pdf

República de Colombia. Ministerio Nacional de Educación. (s.f.). Estándares básicos de competencias en Lenguas Extranjeras: inglés. Recuperado de https://www.mineducacion.gov.co/1759/ articles-339975_recurso_7.pdf

Samper, J. y Maussa, E. (2014). Desarrollo moral y competencias ciudadanas en la juventud universitaria. Jurídicas CUC, 10 (1), 43 - 60.

Zapata, L. (2011). Factores académicos asociados al bajo rendimiento en inglés en las pruebas ECAES presentadas por los estudiantes de la Facultad de Educación en el año 2009. [Tesis de pregrado]. Fundación Universitaria Luis Amigó, Medellín, Colombia. 